\title{
Contextos formais de aprendizagem de treinadores e treinadoras de nível nacional e internacional de goalball
}

\author{
Formal learning contexts of coaches at the national and international level of goalball \\ Contextos formales de aprendizaje de entrenadores y entrenadoras de goalball en nacional e internacional
}

Alessandro Tosim

Universidade Estadual de Campinas - FEF, Brasil

alessandrotosin@hotmail.com

(DD https://orcid.org/0000-0003-2901-8493

Larissa Rafaela Galatti

Universidade Estadual de Campinas - FCA, Brasil

lgalatti@unicamp.br

(iD https://orcid.org/0000-0003-1743-6356

\section{Paulo César Montagner}

Universidade Estadual de Campinas - FEF, Brasil

cesar.montagner@fef.unicamp.br

(iD https://orcid.org/0000-0002-5764-8022

\section{Resumo:}

Este estudo analisou a formação de treinadores de nível nacional e internacional de goalball quanto aos contextos formais de aprendizagem. A amostra foi composta por 81 treinadores esportivos de goalball provenientes de 23 países e foi dividida em dois grupos: o primeiro composto por 47 Treinadores de Nível Nacional (TNN) e o segundo composto por 34 Treinadores de Nível Internacional (TNI). Para a análise das questões de múltipla escolha, foi utilizada estatística descritiva com média \pm D.P. Dentre os resultados encontrados, destacamos: relacionado ao gênero, $76,5 \%$ dos TNN e $85 \%$ TNI são homens. Sobre os contextos formais de aprendizagem, 68\% dos TNN e TNI são graduados em Educação Física e 16\% TNN e TNI em áreas afins. Relacionado com o tempo de experiência, os treinadores internacionais tem uma média 5 anos mais de experiência que os nacionais. Concluímos que o contexto formal se mostra relevante na formação de treinadores de goalball nos dois níveis investigados.

PALAVRas-CHAVE: Esporte paralímpico, Formação profissional, Treinador esportivo.

\section{Abstract:}

This study analyzed the training of national and international goalball coaches, regarding formal learning contexts. The sample consisted of 81 goalball coaches from 23 countries and was divided into two groups: the first composed of 47 National Level Coaches (NNT) and the second one composed by 34 International Level Coaches (TNI). For the analysis of the multiple choice questions, descriptive statistics with mean \pm SD were used. Among the results found, we highlight: related to gender, $76.5 \%$ of TNN and $85 \%$ TNI are male. Regarding formal contexts and mediated learning situations, 68\% of TNN and TNI are graduates in Physical Education and 16\% TNN and TNI in related areas. Related to the length of experience, national coaches have an average of 8 years while international coaches have an average of 13 years. We conclude that the formal context is relevant in the formation of goalball coaches in the two investigated levels.

KEYwORDS: Paralympic sport, Coach education, Sport coach.

\section{RESUMEN:}

Este estudio analizó la formación de entrenadores de goalball nacionales e internacionales, en contextos formales y situaciones de aprendizaje mediado. La muestra estuvo conformada por 81 entrenadores deportivos de goalball de 23 países y se dividió en 
dos grupos: el primero compuesto por 47 entrenadores de nivel nacional (NNT) y el segundo compuesto por 34 entrenadores de nivel internacional (TNI). Para el análisis de las preguntas de opción múltiple se utilizó estadística descriptiva con media \pm DE, entre los resultados encontrados destacamos: relacionados con el género, $76,5 \%$ de TNN y $85 \%$ TNI son hombres. En cuanto a contextos formales y situaciones de aprendizaje mediado, el 68\% de TNN y TNI son graduados en Educación Física y el 16\% de TNN y TNI en áreas afines. En relación con la duración de la experiencia, los entrenadores nacionales tienen un promedio de 8 años mientras que los entrenadores internacionales tienen un promedio de 13 años. Concluimos que el contexto formal es relevante en la formación de entrenadores de goalball en los dos niveles investigados.

Palabras clave: Deporte paralímpico, Formación profesional, Entrenador deportivo.

\section{INTRODUÇÃo}

O Goalball é um esporte criado em 1946 com o intuito de reabilitar soldados que sofreram lesões relacionadas ao órgão da visão no período de guerra. Configura-se como um jogo esportivo coletivo (JEC) sem invasão territorial disputado entre duas equipes com jogadores que apresentam deficiência visual ${ }^{1}$, sendo inserido nos Jogos Paralímpicos em 1976 de Toronto, somente com a categorias masculina, e em 1980, na Holanda, o feminino (Furtado, Cunha, Morato, Michael, \& Gutierrez, 2016; Molik et al., 2015; Morato, Simões, \& Almeida, 2012; Scherer, Karasiak, \& Petroski, 2012; Tosim et al., 2008).

A modalidade apresenta características fisiológicas semelhantes a outros jogos esportivos coletivos, sendo um esporte acíclico, tendo como metabolismo predominante o aeróbio e determinante o anaeróbio alático, exigindo elevado nível de potência muscular, por suas ações explosivas e ocorrerem em tempos menores que 10 segundos (Alves et al., 2018).

Atualmente é praticado em mais de 94 países do mundo e no Brasil não é diferente, já que temos aproximados 80 clubes cadastrados e competindo os campeonatos regionais, estaduais e nacionais, organizados pela Confederação Brasileira de Desportos de Deficientes Visuais (CBDV, 2020).

Um dos responsáveis pela evolução da modalidade é o treinador esportivo, com concepções do esporte sendo modificadas a partir da busca da eficácia nas metodologias associadas a estratégia, tática, técnica e física no treinamento, introdução de equipes multidisciplinares no processo de preparação, acompanhamento do analista de desempenho nos treinamentos, passando informações relevantes para o desempenho da equipe, participação com mais frequência em competições internacionais e profissionalização dos atletas, são fatores determinantes para evolução do esporte no Brasil, sendo atualmente uma potência mundial nas categorias masculina e feminina.

A função de treinador esportivo é multifacetada e altamente complexa, atrelada a relações de poder, em que seu desempenho está largamente influenciado pelo contexto em que está envolvido (Potrac, Jones, \& Armour, 2002). Estudos relacionados ao treinador esportivo no Brasil ficaram mais evidenciados a partir de 2009 (Galatti et al., 2016). No cenário internacional, países como Canadá, Austrália, Reino Unido e Estados Unidos apresentam números significativos relacionado à produção científica associada ao treinador esportivo (Trudel, Gilbert, \& Werthner, 2010).

Côté e Gilbert (2009) apontam três âmbitos de conhecimentos essenciais atrelados ao treinador esportivo. $\mathrm{O}$ conhecimento profissional diz respeito às características técnico-táticas da modalidade; o conhecimento interpessoal, que remete às relações estabelecidas entre as pessoas, o convívio social e a capacidade de comunicação; e o conhecimento intrapessoal, que agrega elementos de autoconhecimento, capacidade de reflexão, introspecção e ética pessoal.

Ao investigar o treinador esportivo, é importante destacar que a formação desses indivíduos ocorre em diferentes formas. Autores como Cushion \& Lyle (2010), Cushion et al. (2010), Jiménez, Lorenzo e Gómez (2009), Mallet et al.(2009) descrevem que a formação do treinador esportivo está pautada em diferentes fontes de conhecimento e contextos, sendo destacado neste artigo o formal, que é aquele caracterizado pela formação institucionalizada, estruturada hierarquicamente, apresentando ao seu término certificações. 
Estudos tem sinalizado que o contexto formal contribui com conhecimentos teóricos e filosóficos de base, mas apresenta déficits em abordar aspectos práticos do treinador esportivo, o que é essencial no percurso formativo de treinadores (Cushion, Armour, \& Jones, 2003; Gilbert \& Trudel, 2001, Milistetd et al., 2017).

No caso brasileiro, treinadores de goalball devem cursar graduação em Educação Física e filiar-se ao Conselho Federal de Educação Física (CREF, 2001) para exercer a função em qualquer cenário de atuação profissional, seja na iniciação esportiva, participação e equipes de alto rendimento, conforme a Lei 9696/98 2 (Brasil, 1998). Esta formação possibilita ao treinador esportivo ter conhecimentos básicos nas áreas biológicas (anatomia, fisiologia do exercício, biomecânica do movimento) entre outras e na área pedagógica (pedagogia dos esportes individuais e coletivos, iniciação e treinamento esportivo) além de outras disciplinas que contemplem a formação do treinador esportivo. Em outros países, como no Canadá por exemplo, a formação de treinador esportivo ocorre por meio da Associação de Treinadores do Canadá (Coaching Association of Canadá - CAC), credenciada para formar treinadores esportivos. Os modelos de formação fazem parte de um programa formal de aprendizagem, desenvolvidos por entidades regulamentadas, que concedem certificações para exercer a profissão de treinador esportivo (Milistetd et al., 2017). Considerando os profissionais de diferentes países, que se formaram treinadores sob legislações distintas, emerge a pergunta que originou este estudo: será que treinadores que atuam em nível nacional e aqueles que atuam em nível internacional tem perfil diferente de formação formal?

Relacionado ao desenvolvimento profissional dos treinadores esportivos paralímpicos, indivíduos estes voluntários ou profissionais, que buscam êxito nas principais competições estaduais, nacionais ou internacionais das modalidades paralímpicas, vem passando do orientador técnico do esporte adaptado na década passadas ao profissional especializado do esporte paralímpico. Atualmente, o treinador esportivo paralímpico para alcançar o êxito, precisa se transformar e processos formativos são importantes. Treinadores do esporte paralímpico, apresentam particularidades relacionadas ao processo de treinamento de atletas com deficiência (Facundo et al., 2019).

O treinador esportivo paralímpico atual, procura a busca do rendimento esportivo da pessoa com deficiência (PCD), transformando o conceito reabilitação em performance de atletas esportivos, desenvolvendo atletas que tem como característica a deficiência por meio da evolução física, técnica e tática, utilizando-se de metodologias do treinamento esportivo que na maioria das vezes são adaptados do esporte olímpico ou já elaborados para o contexto paralímpico, evidenciando que o esporte paralímpico está cada vez mais exigente no sentido de que o atleta paralímpico passe a ser visto com potenciais e capacidades similares ou até maiores que o atleta olímpico, porém com uma deficiência.

O goalball é uma modalidade desenvolvida para atletas com deficiência visual, tendo métodos próprios de treinamento. Para isto, a qualidade e quantidade de treinadores esportivos é um fator determinante para cada nível do continuum de desenvolvimento do esporte (Winand, 2010; Taylor, Werthnet, \& Culver, 2014). Sendo assim, o objetivo do estudo foi analisar a formação de treinadores de nível nacional e internacional de goalball, quanto aos contextos formais de aprendizagem.

\section{Metodologia}

\section{Participantes}

Fizeram parte do estudo 81 treinadores de goalball, em amostra dividida em dois grupos: o primeiro grupo foi composto por 47 Treinadores de Nível Nacional (TNN) que participaram de competições de nível escolar, regional, estadual e nacional e o segundo composto por 34 Treinadores de Nível Internacional (TNI) que participaram de competições de nível continental, mundial e Jogos Paralímpicos. Como caracterização, 41 
são brasileiros (de 14 diferentes estados) e 40 estrangeiros, dos quais 22 são do continente Americano, 12 do continente Europeu, três do continente Asiático, 2 da Oceania e um do continente africano.

O continente americano predominou na amostra do estudo. Isto aconteceu em decorrência do pesquisador principal ser do continente e ter maior acesso a estes treinadores, já que ministrou várias oficinas e participou de competições continentais em alguns países deste continente. O continente asiático se mostrou com baixo número de treinadores, já que países como China e Japão, potências na modalidade, não participaram do estudo e poderiam contribuir ainda mais para os resultados; as ausências se justificam pela dificuldade em encontrar respondentes com conhecimento da língua inglesa e pela China especificamente, não ter acesso aos arquivos da plataforma Google. No entanto, vale destacar que pesquisas envolvendo treinadores de 5 continentes não são frequentes, o que demonstra uma rica amostragem para apresentação final deste estudo.

\section{Procedimentos}

Os treinadores de goalball receberam em seus e-mails o link para acesso a plataforma Google Forms, nos idiomas português, espanhol ou inglês, que ao acessá-lo o direcionava ao questionário. $\mathrm{O}$ documento, na sua introdução, apresenta todas as recomendações do estudo e os voluntários assim que aceitassem participar, autorizavam automaticamente o Termo Livre e Esclarecimento.

Para o desenvolvimento da pesquisa, este projeto foi submetido e aprovado pelo Comitê de Ética e Pesquisa da Universidade Estadual de Campinas - UNICAMP, CAEE 11272719.1.0000.5404.

\section{Resultados e discussão}

Iniciamos por apresentar a caracterização dos TNN e TNI, o que já evidencia alguns diferenciais: TNI apresentaram maior média de anos de experiência prática (13 anos) em relação aos TNN ( 8 anos): conforme expressa a Figura 1:

Figura 1: Tempo de experiência prática em relação aos treinadores.

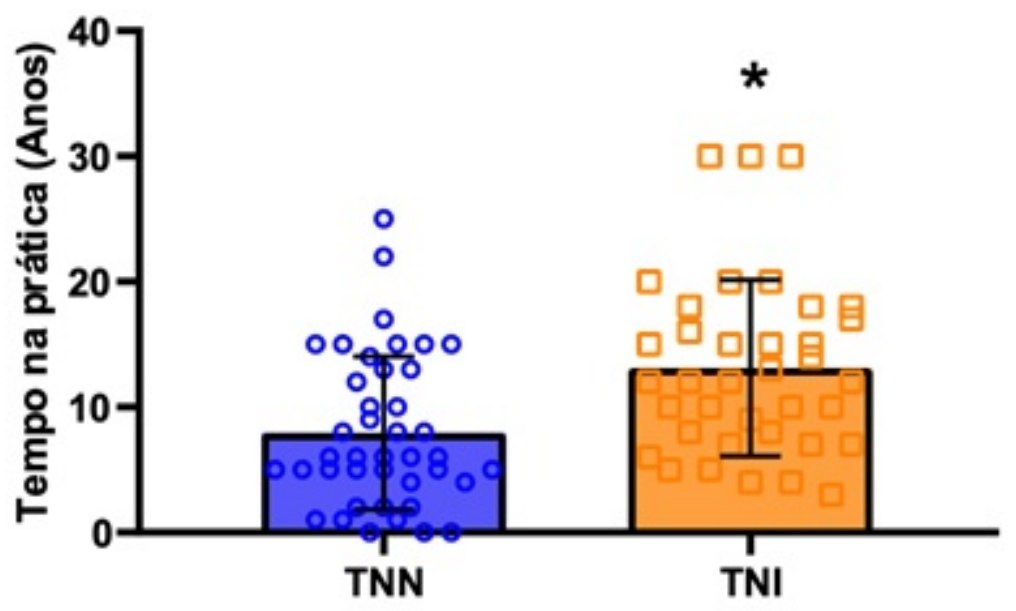

Nota: TNN - Treinadores de Nível Nacional; TNI - Treinadores de Nível Internacional; * $\mathrm{p} \leq 0,05$ Fonte: Elaboração própria

Relacionado ao gênero, encontramos a predominância do masculino com 76,5\% dos TNN e 85\% TNI. O Comitê Olímpico Internacional relata que a equidade de gênero é um componente crítico da administração esportiva e apoia o aumento de mulheres inseridas no sistema esportivo (Comitê Olímipico Internacional [COI], 2014). De um total de 259 federações esportivas nacionais com 22 modalidades esportivas 
diferentes, $71,4 \%$ não apresentam mulheres cadastradas como treinadoras esportivas (Ferreira et al., 2013). Historicamente, o esporte é uma instituição masculina, não só em números, mas também por apresentar valores e normas de comportamento que promovem e naturalizam a ideia de superioridade masculina, tanto nos espaços físicos destinado aos esportes (campos, quadras, pistas, entre outros) como nas hierarquias organizacionais. Por meio do esporte, os homens reafirmam os padrões de privilégio e a subordinação das mulheres que existe fora da realidade esportiva (Whitson, 1990).

As mulheres tendem a ter mais oportunidades de serem treinadoras em modalidades consideradas de menor prestígio e naqueles tradicionalmente femininos (Kamphoff, Armentrout, \& Driska, 2010). Ao limitar as mulheres a serem treinadoras apenas de esportes tradicionalmente femininos consiste em uma maneira de minimizá-las e com isto manter a hegemonia masculina (Reade, Rodgers, \& Norman, 2009). O esporte para pessoas com deficiência e o paralímpico, por ter por princípio proporcionar oportunidades para todos, é um caminho para potencializar o aumento de mulheres no sistema esportivo, nas funções de treinadoras, auxiliares, fisiologistas, preparação física e também em setores auxiliares aos esportes, como nutrição, psicologia e fisioterapia. Além disto, muitas das vezes as atletas com deficiência necessitam de suportes para seus cuidados pessoas, sendo conveniente que tenha mulheres nas comissóes técnicas para auxilia-las. Quanto à formação em contexto formal dos treinadores de nível nacional encontramos o descrito na Tabela 1:

\begin{tabular}{|c|c|c|c|c|c|c|c|}
\hline & CONTINENTE & PAis & GENERO & FORMAÇÃO & AREA & INSTITUIÇÃO & POS GRADUACุÃO \\
\hline $\begin{array}{l}\mathrm{T} \\
\mathrm{N} \\
\mathrm{N}\end{array}$ & Americano & $\begin{array}{c}\text { Brasil }=32 \\
\text { Venezuela }=01 \\
\text { Chile }=10 \\
\text { Colombia }=02\end{array}$ & $\begin{array}{l}M=35 \\
F=10\end{array}$ & $\begin{array}{l}\text { Superior completo }=44 \\
\text { Médio Incompleto }=01\end{array}$ & $\begin{array}{c}\text { Educaçào Fisica }=36 \\
\text { Áreas a fins }=05 \\
\text { Educaçăo Integral }=01 \\
\text { Administraçăo }=01 \\
\text { Programação }=01\end{array}$ & $\begin{array}{l}\text { Pública }=18 \\
\text { Privada }=26\end{array}$ & $\begin{array}{c}\text { Doutorado }=03 \\
\text { Mestrado }=06 \\
\text { Especializaçăo }=24\end{array}$ \\
\hline $\begin{array}{l}\mathrm{T} \\
\mathrm{N} \\
\mathrm{N}\end{array}$ & Oceania & Australia $=01$ & $\mathrm{M}=01$ & Superior Incompleto $=01$ & - & - & - \\
\hline $\begin{array}{l}\mathrm{T} \\
\mathrm{N} \\
\mathrm{N}\end{array}$ & Europa & Portugal $=01$ & $F=01$ & Médio completo $=01$ & - & - & - \\
\hline $\begin{array}{l}\mathrm{Re} \\
\text { sul } \\
\text { tad } \\
\text { os }\end{array}$ & & $\begin{array}{l}95 \% \text { continente } \\
\text { americano }\end{array}$ & $\begin{array}{l}76,5 \% \mathrm{M} \\
23,5 \% \mathrm{~F}\end{array}$ & $93,5 \%$ Superior completo & $76,5 \%$ Educação Fisica & $55 \%$ Privadas & $51 \%$ Especialização \\
\hline
\end{tabular}

Nota: TNN - Treinadores de Nível Nacional Fonte: Elaborado pelos autores

Para a formação em contexto formal de treinadores de nível internacional, encontramos o descrito na Tabela 2: 
Tabela 2: Contexto formal de aprendizagem dos TNI

\begin{tabular}{|c|c|c|c|c|c|c|c|}
\hline & CONTINENTE & PAIS & GENERO & FORMAÇAO & AREA & INSTITUIÇAO & POS GRADUACCAO \\
\hline $\begin{array}{l}\mathrm{T} \\
\mathrm{N} \\
\mathrm{I}\end{array}$ & Americano & $\begin{array}{c}\text { Brasil }=09 \\
\text { Costa Rica }=01 \\
\text { Colombia }=01 \\
\text { Argentina }=02 \\
\text { Peru }=02 \\
\text { Canada }=01 \\
\text { EUA }=02\end{array}$ & $\begin{array}{l}M=16 \\
F=02\end{array}$ & $\begin{array}{c}\text { Superior completo }= \\
18\end{array}$ & Educação Fisica $=12$ & $\begin{array}{l}\text { Püblica }=14 \\
\text { Privada }=05\end{array}$ & $\begin{array}{c}\text { Mestrado }=05 \\
\text { Especialização }=06\end{array}$ \\
\hline $\begin{array}{l}\mathrm{T} \\
\mathrm{N} \\
\mathrm{I}\end{array}$ & Oceania & Australia $=01$ & $M=01$ & $\begin{array}{c}\text { Superior completo }= \\
01\end{array}$ & $\begin{array}{c}\text { Business e Marketing = } \\
01\end{array}$ & Pública $=01$ & - \\
\hline $\begin{array}{l}\mathrm{T} \\
\mathrm{N} \\
\mathrm{I}\end{array}$ & Europa & $\begin{array}{c}\text { Portugal }=01 \\
\text { Espanha }=02 \\
\text { Alemanha }=01 \\
\text { Russia }=01 \\
\text { Lithuania }=02 \\
\text { Belgica }=01 \\
\text { Grécia }=01 \\
\text { Turquia }=01 \\
\text { Suécia }=01\end{array}$ & $\begin{array}{l}M=09 \\
F=02\end{array}$ & $\begin{array}{l}\text { Superior completo = } \\
\quad 09 \\
\text { Superior Incompleto } \\
=02\end{array}$ & $\begin{array}{c}\text { Educação Fisica }=03 \\
\text { Esportes }=03\end{array}$ & Pública $=09$ & $\begin{array}{c}\text { Doutorado }=01 \\
\text { Mestrado }=02 \\
\text { Especialização }=03\end{array}$ \\
\hline $\begin{array}{l}\mathrm{T} \\
\mathrm{N} \\
\mathrm{I}\end{array}$ & Africa & Egito & $\mathrm{M}=01$ & $\begin{array}{c}\text { Superior completo }= \\
01\end{array}$ & Educação Fisica = 01 & Pública = 01 & Especialização $=01$ \\
\hline $\begin{array}{l}\mathrm{T} \\
\mathrm{N} \\
\mathrm{I}\end{array}$ & Asia & Israel & $\begin{array}{l}M=02 \\
F=01\end{array}$ & $\begin{array}{c}\text { Superior completo }= \\
03\end{array}$ & Educacào Fisica $=03$ & $\begin{array}{l}\text { Pública }=01 \\
\text { Privada }=02\end{array}$ & $\begin{array}{c}\text { Mestrado }=01 \\
\text { Especialização }=02\end{array}$ \\
\hline Resultados & 5 Continentes & $\begin{array}{l}55 \% \text { Continente } \\
\text { americano }\end{array}$ & $85 \% \mathrm{M}$ & $\begin{array}{l}94 \% \text { Superior } \\
\text { completo }\end{array}$ & $56 \%$ Educaçào Fisica & $76 \%$ Públicas & 35\% Especialização \\
\hline
\end{tabular}

Nota: TNI - Treinadores de Nível Internacional. Fonte: Elaborado pelos autores

Analisando os contextos formais de aprendizagem, 55 (68\%) dos TNN e TNI são graduados em Educação Física e 13 (16\%) TNN e TNI em áreas afins como esportes, Pedagogia em Educação Física, Deportes, Superior em Preparação Física, Ciência do Movimento Humano, Metodologia do Entrenamento Deportivo e Biomecânica. Entre os TNN, a maioria é graduada em Educação Física. Esse resultado pode ter influência da prevalência de brasileiros na amostra, já que no Brasil a atuação como treinador está sob o guarda-chuva da regulamentação profissão de Educação Física é regulamentada, em cumprimento ao que determina a Lei Federal no 9696 , de $1^{\circ}$ de setembro de 1998 (Brasil, 1998).

A Lei brasileira no 9696/98, que regulamentou essa profissão, é prerrogativa do profissional graduado em Curso Superior de Educação Física (Licenciatura ou Bacharelado), com registro no Sistema CONFEF/ CREFs. Portanto, trata-se de um campo profissional legalmente organizado, integrado a área da saúde e da educação (Conselho Federal de Educação Física [CONFEF], 2001). No Parecer CNE/CES 215/1987 e na Resolução CNE/CES 03/1987, diferentemente dos documentos anteriores, são contemplados a Educação Física especial na formação dos professores de Educação Física, fazendo parte do grupo das disciplinas técnicas na graduação (BRASIL, 2009). Portanto, cursos de graduação em Educação Física no Brasil apresentam em sua grade curricular disciplinas especificas para pessoa com deficiência, abordando a atividade motora e o esporte para pessoas com deficiência. 
Nos outros países analisados, não encontramos nenhum que apresente uma regulamentação específica para a profissão, mas observamos um número significativo de treinadores com graduação em Educação Física ou áreas afins. Isso mostra a importância da área para o desenvolvimento do esporte e a aprendizagem adquirida por meio do ensino formal, que é multifacetado, mas contribui com este treinador mais preparado para suas ações, que atrelada a oportunidades em contexto não formais e informais fazem um treinador completo para a modalidade esportiva. Por não apresentarem nenhuma regulamentação para a área, qualquer pessoa pode atuar como treinador esportivo especialmente em níveis iniciais, e geralmente as Federações Esportivas e as Organizações Nacionais de Esporte têm sido responsáveis pela formação destes treinadores em diversos países (International Council For Coaching Education [ICCE], 2013). Nestes programas de formação, os treinadores são certificados para atuar em diferentes contextos esportivo como: participação, lazer, educação, saúde e rendimento (Côte \& Gilbert, 2009). Como exemplo, citamos o Canadá, onde a Associação de Treinadores do Canadá (Coaching Association of Canadá - CAC) é a entidade responsável pela formação formal de treinadores esportivos, fornecendo as certificações necessárias para o treinador exercer sua profissão (Werthner, Culver, \& Trudel, 2012).

O ensino superior é um importante momento para a formação destes treinadores esportivos, pois possibilita conhecimento específico da Educação Física ou áreas afins, especialmente por proporcionar um elevado corpo de conhecimentos, recursos e possibilidades para impulsionar a aprendizagem destes profissionais (Banack, Bloom, \& Falcão, 2012; Jones, Morgan, \& Harris, 2012). No caso do esporte paralímpico, sua relevância parece ser ainda maior, pois muitos treinadores vão conhecer essa possibilidade de atuação somente na graduação (Tosim et al., 2020). Diante desta discussão, acreditamos que no Brasil, por apresentar uma regulamentação com regras e obrigatoriedades para a profissão, ganha credibilidade para atuação no campo da Educação Física, com conhecimentos necessários, ainda que por vezes pouco específicos, para atuação de treinador esportivo.

Disciplinas que proporcionam os conhecimentos básicos nas áreas biológicas (anatomia, fisiologia do exercício, biomecânica do movimento), sociológica (sociologia do esporte) e na área pedagógica (pedagogia dos esportes individuais e coletivos, iniciação e treinamento esportivo) além de outras disciplinas que complementam a formação do profissional, dão a base para estes futuros treinadores, mas a complementação com o conhecimento não formal e o informal se fazem necessários para se tornar um treinador completo.

Os cursos de graduação, por serem generalistas, podem ter levado um número significativo de TNN a realizar pós-graduação nível latu sensu (especialização) na área da Educação Física e do esporte para pessoas com deficiência, com o intuito de estar mais preparado para atuar com este público. Já os TNI apresentam resultados significativos na pós-graduação nível stricto sensu (mestrado e doutorado), já que muitos países não apresentam cursos latu sensu na sua formação complementar. Observamos também que dos 7 TNN ( 4 mestrados e 3 doutorados) e 9 TNI ( 8 mestrados e 1 doutorado) realizaram seus cursos de pós-graduação nível stricto senso em universidades públicas e apenas 1 TNN apresentou doutorado em universidade privada. Diante destes dados, políticas de desenvolvimento dos cursos de pós-graduação nível stricto senso são necessárias e podem contribuir com o desenvolvimento não apenas de treinadores, mas do esporte paralímpico de maneira geral.

Botomé (1998) afirma que o desenvolvimento de um país necessita de otimização dos seus processos de produção do conhecimento e de maximização do acesso ao conhecimento disponível para sua população. Destacamos que ambos os cursos vão perdurar ao longo da vida e não se restringem apenas a dimensão pragmática de preparação direta para o trabalho ou para ascensão na carreira, como costumeiramente é relatado pelo senso comum. A proposta destes cursos é ir além da dimensão formativa do educando, buscando preparar o indivíduo para o exercício da cidadania, que transcende o limite do campo profissional (Fonseca \& Fonseca, 2016). 


\section{Conclusões}

Os resultados desse estudo contribuem em evidenciar que o contexto formal de aprendizagem parece ser significativo para treinadores de goalball, já que a grande maioria dos TNN e TNI possuem graduação em Educação Física ou áreas a fins. Treinadores que alcançaram nível internacional tem mais anos de experiência que os nacionais, indicando que a experiência prática contribuir para alcançar esse nível de atuação. Atletas do esporte paralímpico atualmente querem e passam a ser reconhecidos por seus feitos atléticos e treinadores com bases científicas a partir da graduação em Educação Física e áreas afins contribuem na luta luta anticapacitista.

Algumas limitações devem ser reconhecidas: a prevalência de treinadores do Brasil e Américas e baixa presença de treinadores de outros continentes, por dificuldades de acesso ou no contato com os idiomas assumidos no estudo (português, inglês e espanhol). Outro fator a considerar é a respeito do número de treinadoras mulheres, são poucas as que atuam com o goalball em nível nacional e internacional, necessitando que as federações e comitês paralímpicos nacionais e internacionais desenvolvam políticas de fomento para ampliar este quadro.

\section{REFERÊNCIAS}

Alves, I. S., Kalva Filho, C. A., Aquino, R., Travitzki, L., Tosim, A., Papoti, M., \& Morato, M.P. (2018). Relationships Between Aerobic and Anaerobic Parameters with Game Technical Performance in Elite Goalball Athletes. Frontiers in Physiology, 9. https://doi.org/10.3389/fphys.2018.01636/full

Banack, H., Bloom, G., \& Falcão, W. (2012). Promoting long term athlete development in cross country skiing through competency-based coach education: a qualitative study. International Journal of Sports Science \& Coaching, 7(2), 301-316. https://doi.org/doi:10.1260/1747-9541.7.2.301

Botomé, S. P. (1998). Qualificação de cientistas e professores de nível superior para o desenvolvimento científico, tecnológico e universitário do país por meio de mestrados e doutorados descentralizados: avaliação de uma experiência, Educação Brasileira, 20(41), 49-77.

Brasil (1998). Lei 9696. Regulamentação da Profissão de Educação Física. Diário oficial da União. Retrieved Outubro 21, 2021, de http://www.planalto.gov.br/ccivil_03/leis/19696.htm

Brasil. (2009). Conselho Nacional de Educação. Resolução CNE/CP n. 4, de 6 de abril de 2009.

Comitê Olimpico Internacional COI. (2014). Factsheet women in the olympic movement. Recuperado em 01 de fevereiro de 2020, de http://www.olympic.org/Documents/Reference_documents_Factsheets/Women_in_O lympic_Movement.pdf

Confederação Brasileira de Desportos de Deficientes Visuais CBDV. Estatuto CBDV. (2020). Recuperado de https:/ /www.dropbox.com/s/mj52ytf7ham9euc/Estatuto\%20CBDV\%202020.pdf?dl=0

Conselho Federal de Educação Física - CONFEF. (2001). Documento de intervenção do Profissional de Educação Física. Recuperado em 10 agosto 2020, de https://www.confef.org.br/confef/conteudo/1705.

Côté, J. (2006). The development of coaching knowledge. International Journal of Sports Science e Coaching, 1(3), 217-222. https://doi.org/10.1260/174795406778604609

Côté, J. \& Gilbert, W. D. (2009). An Integrative Definition of Coaching Effectiveness and Expertise. International Journal of Sports Science e Coaching, 4(3), 307-323. https://doi.org/10.1260/174795409789623892

Cushion, C., Nelson, L., Armour, K., Lyle, J., Jones, R., Sandford, R., \& O’Callaghan, C. (2010). Coach learning and development: A review of literature. Leeds: Sports coach UK.

Cushion, C. \& Lyle, J. (2010). Conceptual development in sports coaching. In: J. Lyle \& C. Cushion (Eds.). Sports coaching: professionalisation and practice (pp. 1-13). London: Elsevier.

Cushion, C., Armour, K. \& Jones, R. (2003). Coach education and continuing professional development: experience and learning to coach. Quest, London, 55(3), 215-230. https://doi.org/10.1080/00336297.2003.10491800 
Facundo, L. C., Mello, M. T., Simim, M.A.M., Duarte, T., Cruz, A.A.S., Narciso, F.V., Ramos, R.A.A., Costa, A.M. \& Silva, A. (2019). A. Trajetória profissional de treinadores no contexto do esporte paralímpico. Movimento, 25(e25034), 1-12. https://doi.org/10.22456/1982-8918.87115

Ferreira, H.J., Salles, J. G. C., Mourão, L., \& Moreno, A. (2013). A baixa representatividade de mulheres como técnicas esportivas no Brasil. Movimento, 19(3),103-124. https://doi.org/10.22456/1982-8918.29087

Fonseca, M. \& Fonseca, D. M. (2016). A gestão acadêmica da pós-graduação lato sensu: o papel do coordenador para a qualidade dos cursos. Educação e Pesquisa, 42(1), 151-164. Recuperado de https://www.redalyc.org/articulo .oa?id=29844947011

Furtado, O., Cunha, L. P., Morato, M. P., Michael, P., \& Gutierrez, G. L. (2016). Health-related physical fitness among young goalball players with visual impairments. Journal of visual impairment e blindness, 110(4), 257 - 267. ht tps://doi.org/10.1177/0145482X1611000405

Galatti, L. R., Bettega, O. B., Brasil, V. Z., Sobrinho, A. E . P. S., Bertram, R., Tozetto, A. V. B., Rodrigues, H. A., Collet, C., Nascimento, J., \& Milistetd, M. (2016). Sport Coaching as a Profession in Brazil: An Analysis of the Coaching Literature in Brazil From 2000-2015. International Sport Coaching Journal, 3(3), 316 - 331. https:/ /doi.org/10.1123/iscj.2015-0071

Gilbert, W. \& Trudel, P. (2001). Learning to coach through experience: reflection in Model Youth Sport Coaches. Journal of Teaching in Physical Education, 21, 16-34. https://doi.org/10.1123/jtpe.21.1.16

International Blind Sports Federation IBSA (2019). Classificação B-1, B-2 and. B-3. Recuperado em 23 de dezembro de 2019, de http://www.ibsa.es/rules/rules.html.

International Council For Coaching Education ICCE. (2010) A strategy for the International Council for Coach Education for the period 2010-2015. Recuperado em 25 de novembro de 2019, de https://www.icce.ws/_assets /files/documents/ICCE_strategy_2012.pdf.

Jiménez, S., Lorenzo, A., \& Gómez, M.À. (2009). Medios de formación de los entrenadores expertos en baloncesto. Cultura, Ciencia y Deporte, 4(11), 119-125. https://doi.org/10.12800/ccd.v4i11.141

Jones, R., Morgan, K., \& Harris, K. (2012). Developing coaching pedagogy: seeking a better integration of theory and practice. Sport, Education and Society, 15(2), 1-17. https://doi.org/10.1080/13573322.2011.608936

Kamphoff, C., Armentrout, S., \& Driska, A. (2010). The Token Female: Women's Experiences as Division I Collegiate Head Coaches of Men's Teams. Journal of Intercollegiate Sport, Champaign, 3(2), 297-315. https://doi.org/10 $.1123 /$ jis.3.2.297

Mallet, C., Trudel, P., Lyle, J., \& Rynne, S. (2009). Formal vs informal coach education. International Journal of Sports Science and Coaching, 4(3), 325-334. https://doi.org/10.1260/174795409789623883.

Milistetd M., Galatti, L. R., Collet, C., Tozetto, A. V. B., \& Nascimento, J. V. (2017). Formação de treinadores esportivos: Orientações para a organização das práticas pedagógicas nos cursos de bacharelado em Educação Física. Journal of Physical Education, 28(1), 1-14. https://doi.org/10.4025/jphyseduc.v28i1.2849

Morato, M. P., Simoes, M. G., \& Almeida, J. J. G. (2012). Os processos de auto-organização no goalball. Revista Brasileira de Ciências do Esporte, 34(3), 741 - 760. https://doi.org/10.1590/S0101-32892012000300015

Molik, B., Morgulec, N. A., Kosmol, A., Perkowski. K., Bednarczuk, G., \& Skowro'nski, W. (2015). Game performance evaluation in male goalball players. Journal of Human Kinetics, 48(1), 43-51. https://doi.org/10. 1515/hukin-2015-0090

Potrac, P., Jones, R., \& Arnour, K. (2002). It's all About Getting Respect': the Coaching Behaviors of an Expert English Soccer Coach. Sport, Education \& Society, 2(7), 183-202. https://doi.org/10.1080/1357332022000018869

Reade, I., Rodgers, W., \& Norman, L. (2009). The Under-Representation of Women in Coaching: A Comparison of Male and Female Canadian Coaches at Low and High Levels of Coaching. International Journal of Sports Science \& Coaching, 4(4), 505-520. https://doi.org/10.1260/174795409790291439

Scherer, R. L., Karasiak, F. C., Silva, S. G., \& Petroski, E. L. (2012). Morphological profile of goalball athletes. Motricidad. European Journal of Human Movement, 28, 1-13. Recuperado de https://www.redalyc.org/articul o.oa?id=274224368001 
Taylor, S., Werthner, P., \& Culver, D. (2014). A case study of a parasport coach and a life of learning. International Sport Coaching Journal, 1(3), 127-138. https://doi.org/10.1123/iscj.2013-0005

Tosim, A., Rodrigues, G. M., \& Mendonça, M. A. B. (2008). Seleção da percepção auditiva e tátil em atletas de goalball em situação defensiva de jogo. Revista Mackenzie de Educação Física e Esportes, 7(3), 181 - 187.

Tosim, A., Marques Filho, C. V., Galatti, L. R., Morato, M. P., Borin, J. P., Montagner, P. C. (2020). Desafios e possibilidades para o goaball brasileiros: concepções a partir do treinador esportivo que atua em clubes nacionais. Pulsar (Jundiai), 12, 64-77. Recuperado de: https://www.researchgate.net/publication/342318072

Trudel, P., Gilbert, W., \& Werthner, P. (2010). Coach education effectiveness. In: J. Lyle \& C. Cushion, (Orgs.). Sports coaching: professionalisation and practice (Cap. 9, pp. 135-152). London: Elsevier.

Trudel, P., Culver, D., \& Werthner, P. (2013). Looking at coach development from the coach-learner's perspective: considerations for coach development administrators. In P. Potrac, W. Gilbert, \& J. Denilson (Eds.). Routledge handbook of sports coaching (pp. 375-387). London: Routledge, 375-387.

Trudel, P., Gilbert, W., \& Rodrigues, F. (2016). The Journey from Competent to Innovator: Using Appreciative Inquiry to Enhance High Performance Coaching. International Journal of Appreciative Inquiry, $18(2)$. https:// doi.org/10.12781/978-1-907549-27-4-5

Werthner, P., Culver, D., \& Trudel. P. (2012). An examination of a large scale coach education program from a constructivist perspective. In R. Schinke. (Ed.) Sport Psychology Insights (pp. 337-354). London, Nova Science Publishers Inc.

Werthner, P. \& Trudel. P. (2006). A new theoretical perspective for understanding how coaches learn to coach. The Sport Psychologist, 20(2), 198-212. https://doi.org/10.1123/tsp.20.2.198

Whitson D. (1990). Sport in the social construction of masculinity. In M. Messner, \& S. Donald. (Eds.). Sport, men and the gender order: critical feminist perspectives (pp. 19-29). Champaign: Human Kineticts.

Winand, M. (2010). The Global Sporting Arms Race. An International Comparative Study on Sports Policy Factors Leading to International Sporting Success (SPLISS). European Sport Management Quarterly, 10(5), 613-615. https://doi.org/10.1080/16184742.2010.524242

\section{Notas}

1 Segundo o manual de classificação da International Blind Sports Federation - IBSA (IBSA, 2016), para as competições oficiais do Goalball, os jogadores devem se enquadrar na classificação esportiva para serem elegíveis para o jogo, sendo esta: Classe B1: da inexistência da percepção de luz em ambos os olhos, ou percepção de luz, porém com incapacidade para reconhecer a forma de uma mão a qualquer distância ou em qualquer direção; Classe B2: da capacidade para reconhecer a forma de uma mão até a acuidade visual de 2/60 (dois por sessenta) e/ou um campo visual menor que 5 (cinco) graus; Classe B3: da acuidade visual superior a 2/60 (dois por sessenta) até uma acuidade visual de 6/60 (seis por sessenta) e/ ou um campo visual maior que 5 (cinco) graus e menor que 20 (vinte) graus.

2 Lei no 9.696 de 01 de Setembro de 1998 Dispõe sobre a regulamentação da Profissão de Educação Física e cria os respectivos Conselho Federal e Conselhos Regionais de Educação Física. Art. 10 O exercício das atividades de Educação Física e a designação de Profissional de Educação Física é prerrogativa dos profissionais regularmente registrados nos Conselhos Regionais de Educação Física. 